\title{
Evaluating the Potential of fNIRS Neuroimaging to Study Engineering Prob- lem Solving and Design
}

\section{Dr. Jacob R. Grohs, Virginia Tech}

Jacob Grohs is an Assistant Professor in Engineering Education at Virginia Tech with Affiliate Faculty status in Biomedical Engineering and Mechanics and the Learning Sciences and Technologies at Virginia Tech. He holds degrees in Engineering Mechanics (BS, MS) and in Educational Psychology (MAEd, $\mathrm{PhD})$.

\section{Dr. Tripp Shealy, Virginia Tech}

Tripp Shealy is an Assistant Professor of Civil and Environmental Engineering at Virginia Tech and principal faculty in the Myers-Lawson School of Construction. He received is doctorate from Clemson University. His research is at the intersection of cognitive psychology and engineering decision making for sustainability.

\section{Darren K. Maczka, Virginia Tech}

Darren Maczka is a Ph.D. candidate in Engineering Education at Virginia Tech. His background is in control systems engineering and information systems design and he received his B.S. in Computer Systems Engineering from The University of Massachusetts at Amherst. He has several years of experience teaching and developing curricula in the department of Electrical and Computer Engineering at Virginia Tech.

Miss Mo Hu, Virginia Tech

Dr. Robin Panneton, Virginia Tech

1981-1985 Ph.D. Developmental Psychology; University of North Carolina at Greensboro, Greensboro, NC 1978-1981 M.A. Developmental Psychology ; University of North Carolina at Greensboro, Greensboro, NC 1974-1978 B.S. PsychologyUniversity of Wisconsin at Milwaukee, Milwaukee, WI

My research interests revolve around issues relevant to infants' perception of information that leads to their emerging communicative skills from birth to toddlerhood. These research questions involve probing aspects of infants' learning about objects, about people, and about themselves in relation to objects and people. I am particularly interested in how multiple sources of dynamic sensory information drive infants' attention to and learning about the world in which they live and their ability to communicate about it.

Mr. Xiao Yang, Virginia Tech, Deparment of Psychology 


\section{Evaluating the Potential of fNIRS Neuroimaging to Study Engineering Problem-solving and Design}

This theory paper introduces functional Near Infrared Spectroscopy (fNIRS) methods to the engineering education community as a research method to augment engineering problem-solving and design studies. As technology develops, we can ask and answer emergent research questions to meet National Science Foundation priorities to understand brain-behavior relationships, specifically in how "collective interactions between brain function and our physical and social environment enable complex behavior" [1]. With researchers already pushing the boundaries of knowledge with teaching, learning, and practice of complex engineering skills, the field of engineering education is well poised to partner with cognitive neuroscientists, developmental psychologists, and others to consider how neuroimaging can complement or supplement pressing research questions.

In the first section of this paper we provide an overview of cognitive neuroscience basics that will enable a broader discussion of salient opportunities and challenges of integrating engineering education and neuroscience research. The second section transitions from the discussion of overarching rationale to a specific focus on engineering problem-solving and design contexts through a brief literature review and suggested areas of future research. In the final section of the paper, we discuss two ongoing exemplar projects, not to report findings, but to offer examples of what research designs could look like and the associated data collection and analysis protocols.

\section{Cognitive Neuroscience Basics}

Simply stated, cognitive neuroscience focuses on empirical data from both human behavior and the brain in order to explore human cognition (thinking, planning, decision making) [2]. The study of behavior has a rich tradition in psychology and is strongly rooted in the primacy of empiricism - that knowledge is built through systematic and objective observation and measurement. A primary goal of empirical study of behavior is generalized causal inference, the notion that A reliably causes B and that such a relationship is reasonably stable across contexts [3]. In aiming towards causal inference, researchers control and manipulate independent variables through experimental design in order to study effects on target dependent variables (e.g, the presentation of a tone causing dogs to salivate because of previous pairing with meat in Pavlov's classical conditioning experiments [4]). In a strict interpretation of studying human behavior, we cannot study cognition directly, instead we can only observe the products of an individual's thinking (e.g., actions taken, answers given to a test, artifacts created). Keeping these limitations in mind, the emergence of techniques to collect data on the brain have revolutionized the study of cognition because they enable the collection of objective measurable physiological data taken between the introduction of a stimulus and the associated response. In essence, that which was once an unexaminable black box can now be examined and in multiple conditions.

In cognitive neuroscience, making sense of brain-behavior relations is a search to understand the functional architectures of cognitive systems [2], [5] - for example, how does some function of interest (e.g., face recognition) occur and in what region? To explore such a question requires a 
model about how the brain works and is organized. Significant bodies of research are built upon the simplifying assumption of modularity to which we might make the analogy of an assembly line in a factory: Each worker performs only one task, and any given function necessarily and reliably involves the same subset of workers. In terms of scientific inquiry, this means that we must merely induce a function (e.g., present the image of former teacher to a participant), identify the specific areas of the brain involved, and we will have reliably characterized the architecture of that function (e.g., face recognition). Further, if we see those areas of the brain involved in any future task then we can reliably assume that the participant is performing the function of interest. Despite evidence that modules are interconnected and do not exhibit the domain specificity of the single-worker single-task model, research anchored in modular brain architecture is still widely accepted. For discussion of a more modern approach to the modularity issue, please see [6]. As imaging techniques and computational power have improved, the modularity assumption can be loosened to instead investigate correlations between modules and identify the networks involved with specific functions [2]. Such a distinction will show up later in the paper as we discuss the difference between univariate and multivariate analyses of brain data.

\section{Brain Data Collection Techniques}

A wide range of technologies are used in collecting brain data though the methods vary greatly according to their spatial resolution, temporal resolution, invasiveness, and cost (see [2] for a good overview). Two common methods used to explore neural processes of decision-making and problem solving under laboratory conditions are electroencephalography (EEG) and functional magnetic resonance imaging (fMRI). However, each have limitations, which has led to an emergent viable third option to study complex processes in more realistic environments, called function near infrared spectroscopy (fNIRS). We will briefly discuss all three methods.

EEG involves a head covering (e.g., cap or net) which places electrodes on the scalp and measures electrical changes in the brain. Temporal resolution is very good (detects quick changes) though spatial resolution is poor because signals often interfere with one another and make it difficult to pinpoint specific brain regions involved in the processing. EEG laboratory equipment is priced such that faculty labs could easily each have their own EEG device (e.g., cost on the order of several hundred dollars). For participants, EEG is minimally invasive as the participant can sit comfortably while wearing the electrodes, though it is important to minimize movement so that electrical signals involved in triggering motion do not interfere with the electrical signals related to the experimental task response. According to Eysneck and Keane [2], EEG methods "are mainly of value when stimuli are simple and the task involves basic processes (e.g., target detection) triggered by task stimuli."

In contrast to EEG measurement of electrical activity, fMRI technology measures activity indirectly through changes in blood flow in the brain. As a brain region is activated, the body sends more blood to that region and fMRI detects these changes by imaging the blood oxygen level-dependent contrast (BOLD) signal in a special magnetic scanner [2]. Because blood flow changes happen over time, the temporal resolution of fMRI is not as good as EEG (i.e. order of seconds compared to milliseconds), but the spatial resolution is very high and thus amenable to 
pinpointing changes within specific regions. fMRI is a very expensive technology and even when an institution purchases a machine, often researchers must pay significant costs per participant (e.g., \$500/hour). Further, data collection can be uncomfortable and constraining as participants must remain still while partially enclosed inside the MRI scanner. fMRI is a leading neuroimaging method currently at the forefront of enhancing what is known about the brain, however, the cost and constraints of the laboratory environment make it difficult to record cognitive processes for extended time and in unrealistic settings during problem-solving and design.

In some ways, fNIRS combines benefits of both EEG and fMRI and is thus extremely interesting as a resource to understand cognition in educational settings. fNIRS uses a series of light sources and detectors through a close-fitting head cap to measure hemodynamic changes in target brain regions. The amount of light detected through the scalp changes as blood flows and the concentration of deoxygenated vs. oxygenated hemoglobin changes (same BOLD response as fMRI). Experimentally, fNIRS machines use a similar simple setup as EEGs so that participants can comfortably sit or stand while wearing a cap that is connected to a data acquisition system. One key advantage is that fNIRS can be used while participants walk, talk, operate a computer, write, or otherwise perform the actions we commonly associate with educational settings. The motion artifacts created by these everyday actions are difficult to control for in both EEG and fMRI methods, but do not pose a significant threat in fNIRS research. Though fNIRS boasts greater spatial resolution than EEG, it lacks the high spatial resolution of fMRI, and thus provides little information about sub-cortical brains regions. It is sufficiently effective, however, to investigate areas such as the prefrontal cortex that are associated with executive function (e.g., planning, problem solving). fNIRS machines are a significant investment (e.g., purchase price of $\$ 50,000-300,000$ ) but at about an order of magnitude of cost less than fMRI, fNIRS is much more amenable to exploratory study if a researcher can find a partner who owns the machine.

\section{Brain Regions}

A thorough discussion of brain regions is out of the scope of this review section. However, because fNIRS is limited to studying cortical regions, it is useful to discuss brain structure for readers to understand the limitations. The cerebral cortex (cortical regions) is the outer surface of the brain and is divided into two mirrored hemispheres, and four lobes that are depicted in figure 1 and briefly described here using descriptions directly from Ormrod in [7]:

- Frontal lobes: "where much of our conscious thinking seems to occur... including language, attention, reasoning, decision making, planning self-regulation, learning strategies, problem solving, consciously controlled movements, and interpretation of other's behaviors"

- Parietal lobes: "receive and interpret somatosensory information - that is, information about temperature, pressure, texture and pain... also actively involved in paying attention, processing word sounds, and thinking about the spatial characteristics of objects and events."

- Occipital lobes: "major responsibility for interpreting and remembering visual information. 
- Temporal lobes: "interpret and remember complex auditory information... also appear to be important in memory for information over the long run"

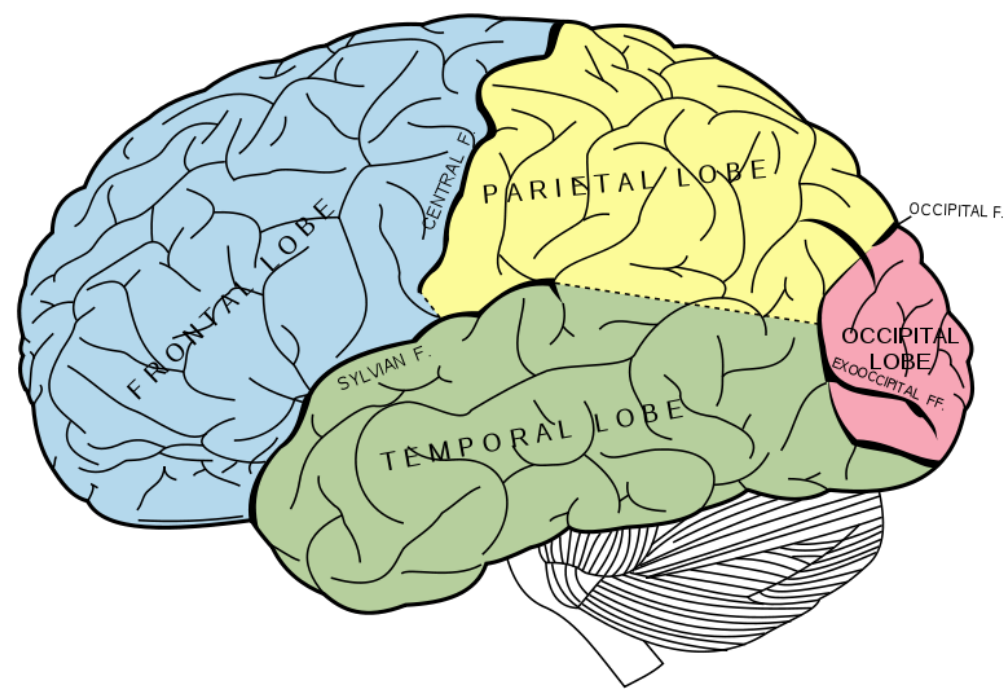

Figure 1:Cerebral cortex image showing four lobes based on Gray's Anatomy of the Human Body [8]

Generally speaking, some functions are more dominantly associated with one hemisphere or the other. For example, language, reading, and math are typically more heavily dependent on the left hemisphere whereas visual and spatial processing are more dependent on the right hemisphere. But, these are broad generalizations as Ormrod reports that "the left hemisphere is the primary language hemisphere for more than $90 \%$ of right-handed individuals but for only about $60 \%$ of left-handed folks." These differences, and the noise created in data interpretation, explains why neuroscience studies often seek only right-handed participants.

One of the primary uses of neuroimaging is to pre-identify regions of the brain that are indicated by the literature informing the research design and then to collect data on that region of interest and also another unrelated area. This is necessary because baseline brain activity even "at rest" is still very active and thus it is best to demonstrate both that a stimulus causes additional activation in a region of interest and that it does not cause additional activation in a nonrelated region. Brodmann's areas [9], [10] are numbered regions which researchers commonly use to describe specific regions within the lobes of the cerebral cortex. These regions are discrete and very well defined.

The connectedness between cortical regions is sparse in the sense that each region only connects with only a few other regions. Three basic functions can be delineated across regions as either primarily responsible for motor, sensory, or association. The frontal cortex, and its many subregions (prefrontal cortex, dorsolateral, ventrolateral) are non-sensory regions specifically related to attention, problem solving and design. In particular, the prefrontal cortex (PFC) is associated 
executive function - planning, decision making, trade-offs, rationalizing future consequences (the associated Brodmann areas include 8, 9, 10, 11, 12, 45, 46, and 47).

\section{Integrating Engineering Education and Cognitive Neuroscience Research through fNIRS} The National Science Foundation has prioritized understanding "cognitive and neural processes in realistic, complex environments" and identified that such research "requires moving beyond static, artificial experimental settings that minimize naturally occurring complexity and interactions" [11]. Drawing from a rich history of social science research, engineering education regularly explores human behavior in realistic, complex environments. For example, when we study how students solve complex problems with both social and technical components, we might analyze artifacts of their work, use verbal think aloud protocols to understand selfreporting of the strategies used, and administer psychometrically validated scales to understand student epistemological beliefs that could be shaping their problem-solving approach. By utilizing accepted qualitative and quantitative methods of social science research, we are able to wade through complexities that often must be controlled for or ignored in laboratory settings. It seems we can contribute much to the collective understanding of brain-behavior relations if we can bring such methods to bear working hand-in-hand with cognitive science experts.

Flipping perspectives, a key limitation of engineering education work often involves the subjectivity and fallibility that comes with participant self-reporting. For example, a student might describe in a think aloud protocol that they easily worked through the necessary steps of the problem without frustration when they might be mistaken, misremembering, or misinforming. Such issues are a key reason that empiricists studying human behavior prioritize directly observable objective evidence of cognition. Yet, the physiological data from neuroimaging provides engineering education researchers with another mode through which to investigate connections - or mismatches - between what participants say, and what they demonstrate. In this sense, engineering education researchers also have much we can learn about our own work by collaborating with cognitive neuroscience experts.

\section{Specific Ways Engineering Education Research Can Be Enhanced with fNIRS}

To understand the degree to which neuroimaging research is being discussed and reported in Engineering Education forums, we conducted a literature search of ASEE PEER proceedings, the Journal of Engineering Education (JEE), and the International Journal of Engineering Education (IJEE) for EEG, fMRI, and fNIRS separately. EEG found 64 hits for ASEE proceedings, 2 hits for JEE, and 3 hits for IJEE. fMRI resulted in 18 hits for ASEE proceedings, 3 hits for JEE, and 3 hits for IJEE. No mentions of fNIRS were found in ASEE proceedings or either journal. Of the hits for EEG or fMRI, none of them were reporting the results of empirical studies that specifically used the method. One pilot study on the "Impact of Cognitive Style and Academic Discipline on Design Prototype Variability" [12] discussed using EEG as part of the pilot but reported no results and instead focused on artifacts from the prototyping activity and the results of survey instruments to measure cognitive style. One paper, "Critical Thinking, Reflective Practice, and Adaptive Expertise in Engineering", had a small section discussing the need for fMRI studies to be conducted while participants "solve problems that are expected to promote critical thinking, reflection, or transfer" but again is not an empirical study. The vast majority of 
remaining hits for EEG or fMRI were discussing engineering instrumentation labs or coursework, signal processing, or in briefly referring to how a finding in neuroscience might support a particular grounding or claim of the paper. Though we did not search outside of ASEE, JEE, and IJEE for this paper, the lack of empirical research combining engineering education with neuroimaging leads us to explore possible fruitful rationale for why such integration would be beneficial.

A noted benefit of cognitive neuroscience is the clear and consistent mappings between events at the neural level and events at the behavioral level and neurobiological function can provide valuable new measurements to advance theories in education. Though, we cannot brainstorm a comprehensive list of such examples in engineering education, rather the list below is meant to illustrate examples within the context of problem-solving and design, both because these domains are considered central to engineering education [13], and because they are contexts within which the authors conduct researcher and so can use specific examples:

1. Contextual Complexities of Engineering Problems-If/how engineers attend to embedded social, cultural, political, and/or ethical complexities in generating solutions to design problem is exceptionally important. While think-alouds and/or analysis of solution artifacts may lend some insights, brain imaging may let us see specifically if and when such complexities are attended to because of the very different areas of the brain that may be involved in these processes.

2. Means to Operationalize Cognitive Load - Cognitive load theory offers relevant explanations for why learning may not occur in many engineering situations. For example, learning Statics requires synthesizing knowledge from geometry, trigonometry, and physics and if requisite pieces (e.g. quickly resolving a vector into components using triangle ratios) are not sufficiently automatic, the new concept might be missed. One measure of the effectiveness of a novel intervention in engineering education would be to note if decreases in cognitive load are seen. Through fNIRS we could design pre/post testing in order to observe both the intensity and duration of activation in particular regions pre/post intervention. For examples, consider [14], [15]

3. Systems vs Linear Thinking - cognitive flexibility means the ability to select, adapt, and combine knowledge and experience in new ways - an essential construct for solving complex and ill-structured problems [16]. Through literature we know which brain regions support which cognitive function (e.g. visual or spatial thinking) [17], but we do not know how these processes coordinate (working memory - prefrontal cortex and visual processing - occipital lobe) [18], or are developed through learning (from novice to expert). By measuring the cognitive activation and location during tasks like mentally drawing connections between objects and knowledge, we can construct a more detailed understanding of the processes and the network coordination between brain regions. Understanding the regions of activation in the brain required for conceptualizing a system is important because we can begin to assess how learning enhances the temporal response (how fast we think) and how learning reduces the cognitive load (the energy required). 
4. Design Thinking-Design decisions about our physical environment shape our comfort, safety, and even ability to learn. Therefore, better understanding of the role of certain brain areas in design decisions across a range of subject groups promises to advance the practice (and teaching) of design. So, the basic questions that link design thinking to cognitive neuroscience are what brain regions are activated as various groups undertake design activities? And two, are activated regions significantly different based on age and type of educational training? At the same time, answering these questions advances cognitive neuroscience by addressing the data collection challenges that arise when extending methods from task-oriented problems to more cognitively complex design challenges that often lack a standardized (discrete) stimulus onset.

Naturally, in dreaming up ways neuroimaging might benefit ongoing engineering education research, it is also easy to immediately start identifying barriers. Described below are a few examples worth noting:

- Institutional Review Board (IRB) Approval for Human Subjects Research - It is easy to expect that with mention of brain imaging the IRB process must be cumbersome. Our experience in receiving IRB approval was surprisingly smooth and required no extra layers of explanation or review above and beyond a typical application. We included language such as "fNIRS technology is safe, portable and noninvasive. fNIRS are worn as a cap and emit light at specific wavelengths $(700-900 \mathrm{~nm})$ into the scalp. The light scatters, and some is absorbed, before reflecting back to the sensor. The hemoglobin $(\mathrm{HbR})$ and Oxy-hemoglobin (HbO2) absorb more light than water and tissue in the brain. The change in density, or Blood Oxygenation Level Dependent (BOLD) response, indicates activated brain regions." Further, we took special care to include this language in consent documents so that participants were also aware.

- Access to the fNIRS machine - the fNIRS machine was purchased on our campus using state funds intended to benefit the whole university and so while priority use is given to the home department/faculty (e.g., Psychology), we are able to use the machine at no extra cost. While this will certainly vary across institutions, because the cost of fNIRS is about an order of magnitude less than an fMRI, exploratory and unfunded research is more feasible with fNIRS. Once purchased there is no additional cost to operate.

- Analysis Software and Techniques-Vendors that sell and support fNIRS machines use a variety of software to collect, clean, and filter data. However, there is a rich collection of open-source tools to help researchers analyze and visualize data (e.g. Homer2: http://homer-fnirs.org/). Once data has been processed, univariate and/or multivariate statistics are used to answer specific research questions of interest. For example, univariate statistics can help determine if a specific repeated stimulus activates a specific region of the brain, or could examine differences in peak activation within a specific region in the brain across two different stimuli, or the same stimuli and two different categories of participants. Multivariate statistics can help identify regions that are activating together (recall the earlier discussion about loosening modularity assumptions and exploring interconnectivity in the brain by examining correlation between modules). A method such as Principal Components Analysis is useful in these situations [19] and 
should be familiar to some educational researchers as it is conceptually similar to the factor analysis methods employed in quantitative scale development.

\section{The Work We Can Do Together}

While there is great benefit to interdisciplinary collaboration, our team quickly noticed some philosophical differences associated with each paradigm of research that align with the sort of concerns discussed by Borrego, and Borrego, Douglas, and Amelink [20], [21]. While good colleagues might easily agree-to-disagree and move through some of the philosophical differences in an interdisciplinary setting (e.g., the role and value of qualitative research), our team has come to believe that laying within the nuances of the paradigm differences is the key to truly studying the brain in the authentically complex environments of the real world. To this point, we will briefly discuss experimental designs.

Drawing both from historical psychological research and conforming to the temporal resolution limitations of fMRI as the current dominant methodology in neuroimaging, experimental design for the cognitive neuroscientist usually involves a commitment to either a blocked or event related design. Blocked designs are so called because different blocks represent different tasks/stimuli related to the independent variable that are repeated several times so that later results might be averaged across like blocks [22]. For example, if examining whether or not participants process lyrics while listening to music, the researcher might design a study where participants listen to 60 seconds of 10 second music clips with lyrics, followed by 60 seconds of rest, followed by 60 seconds of 10 second music clips with no lyrics, and this cycle would be repeated 10 times each. Data would then be averaged together such that the hemodynamic response for all music with lyrics could be compared to the response for all music without lyrics. Such a design assumes that all music with lyrics and all music without lyrics are all sufficiently similar to be considered the same task. An event-related design would randomize the 10 second clips and present them at randomized intervals (e.g., 10 seconds music without lyrics, 3 seconds rest, 10 seconds music with lyrics, 5 seconds rest, 10 seconds music with lyrics, ...). Though data analysis in event-related designs require more advanced statistics knowledge than blocked designs, a key advantage is the ability to identify outliers post-hoc (e.g., if a particular 10 second clip didn't stimulate in the same way as the other 10 second clips it would have been blocked with in a blocked design) as well as efficiency in data collection (e.g., in the blocked design mentions, $1 / 3$ of all participant time would be spend in a resting condition). Despite the many advantages of these methods, they are not representative of realistic environments - for example, an engineering problem is not a simple stimulus and response but rather a lengthy process of thinking, working, evaluating a solution, recognizing and correcting errors, reworking, etc. Contriving stimuli that both fit a blocked or event-related design and which authentically resemble a complex engineering problem is challenging. We must work together to frame new research questions, and new research designs to answer such research questions if we are to meet the call to study neural processes in realistic and complex environments.

\section{Example 1: Insight and Giving Up During Extended Problem Solving in an Educational Game} In an attempt to explore alternate research designs that might generate knowledge about how the brain works in more complex and realistic environments, our team is studying extended problem- 
solving in an educational computer game environment called Contraption Maker (CM). CM is a commercial computer game involving Rube Goldberg inspired puzzles using physics-based interactions among objects. Because of its connection to STEM concepts, free versions are available for educational use along with community-sourced scenarios for specific purposes (ranging from secondary school common core standards to college freshman in physics). In pilot work not using fNIRS [23], we posed a series of in-game puzzles to students, observed their problem solving attempts, and conducted retrospective interviews. Artifacts from the activities were documented through video recordings of the computer screen which allowed us to study the diverse ways in which participants behave as they work towards solutions. To facilitate quick coding of the video data, our team wrote Python scripts using OpenCV tools (computer vision analysis) to automate the video capture analysis and compute variables of interest (e.g., number of puzzles attempts, time elapsed to solution, documented instances of help-seeking behavior). Such work both automates and provides additional nuance about observed problem-solving process and achievement which is a significant improvement to traditional psychology problemsolving experiments still utilized today such as [24], [25]. Retrospective interviews were conducted to explore participant thought processes while solving the puzzles following best practices from [26], [27].

We have recently built upon this pilot work to include fNIRS data collection so that we might answer two specific research questions:

- During extended problem solving, what degree of accuracy can observed behaviors (i.e. insight or giving up) be classified using only participants' BOLD response?

- What are the neural correlates (i.e. associated regions) of insight and giving up during extended problem solving?

\section{Data Collection}

The intent of this paper is to communicate details to help readers understand what is involved with fNIRS, so we describe data collection here in more detail than a typical methods section.

From start to finish, the participant protocol begins with an orientation about fNIRS and researchers answer any questions the participant may have about the method, our IRB approval, or the consent documents. It is explained that fNIRS is safe and non-invasive and that a cap is required to be placed on their head and with grommets that hold a light source and detector. The light shines onto their scalp and the detector reads the amount of light reflected back. After the IRB form is signed a researcher measures the circumference of the participant's head to outfit the appropriate cap size with the light source and detectors. Following the cap measurement, a researcher conducts an intake interview with questions about general problem solving strategies while another researcher begins setting up the appropriately sized fNIRS cap depending on the participant's head measurements. Fitting a cap that has been pre-prepped (i.e., light source and detector cables applied to correct grommets for regions of interest) and calibrating to make sure all sensors are reading well only takes a few minutes. Poor signals during calibration are likely due to dark and/or thick hair and can be remedied by pushing hair out of the way from that specific site. In our experience, it takes on average 20 minutes from beginning intake with the consent process to being ready to begin data collection with the problem solving activities. The only limitation to the length of time of the study while wearing the cap is related to the fatigue and discomfort of the participant. Our experience has been that participants begin to exhibit some signs of mild discomfort (e.g., running their fingers under the cap, fidgeting) if the fNIRS 
data collection portion of the study takes more than 45 minutes. All actions in Contraption Maker and anything spoken by the participant during gameplay (e.g., "aha", "well that didn't work") are captured using video capture recording on the laptop recording and retrospective think aloud interviews [27], [28] are also conducted in-between and after problem solving activities.

The region of interest for data collection using fNIRS is specific to the type of study being conducted, literature of functionality associated with regions of interest, experimental design, and hypothesis. In both this and the next study, fNIRS channels were configured to collect the BOLD response along the dorsolateral prefrontal cortex (dlPFC) (Brodmann areas 9 and 46). The dlPFC is associated with the executive function such as working memory, cognitive flexibility, planning and reasoning. During cap placement on the participants' head, measurements are taken around the circumference of the head and from the temple to the back of the head and from the temple to the forehead. Based on the guidelines provided by the manufacturer (the system at our institution is a NIRx machine) these measurements help ensure the placement more accurately captures the brain regions of interest.

\section{Data Analysis}

The data collected by fNIRS is the change in optical density. The beer-lambert law states that absorbance of a material sample is directly proportional to its thickness (path length). The beerlambert equation is based on water. Thus, a modified beer-lambert law for blood is used to convert the optical density to produce oxygenated and deoxygenated recorded data [29]. The functional image of this is the Blood Oxygenation Level Dependent signal (BOLD), which corresponds to the concentration of oxy and deoxy-hemoglobin.

However, the BOLD response can be influenced by physiological changes in heart rate, instrument noise and experimental error. Thus, pre-process filtering using high and low band pass filters can help correct of these errors. Further, recording a baseline activity before the task begins can be subtracted for the event or block experimental tasks to more narrowly report the change in hemodynamics due to the event or block.

Once the data is filtered and a baseline is subtracted, numerous methods can be used to analyze the data. The most typical methods for analysis include some form of measuring:

1. Delta amplitude

2. Peak amplitude

3. Time to reach peak

4. Slope measurement

5. Area under the curve

Further analysis includes general linear models, especially for block design experiments where the expected response would be an increase in the canonical BOLD function during the trial and a return to homeostatic levels following a rest. As mentioned earlier, others use a multi-variant approach. More still, a recent approach is using Support Vector Machines [30]. For more on data processing methods see [31]. As an example of what multi-channel data looks like, see Figure 2 below which shows an overlay of the video capture from the Contraption Maker study (the participant has clicked the help button for an item) and the processed fNIRS data to show oxygenated and deovygenated hemoglobin. 

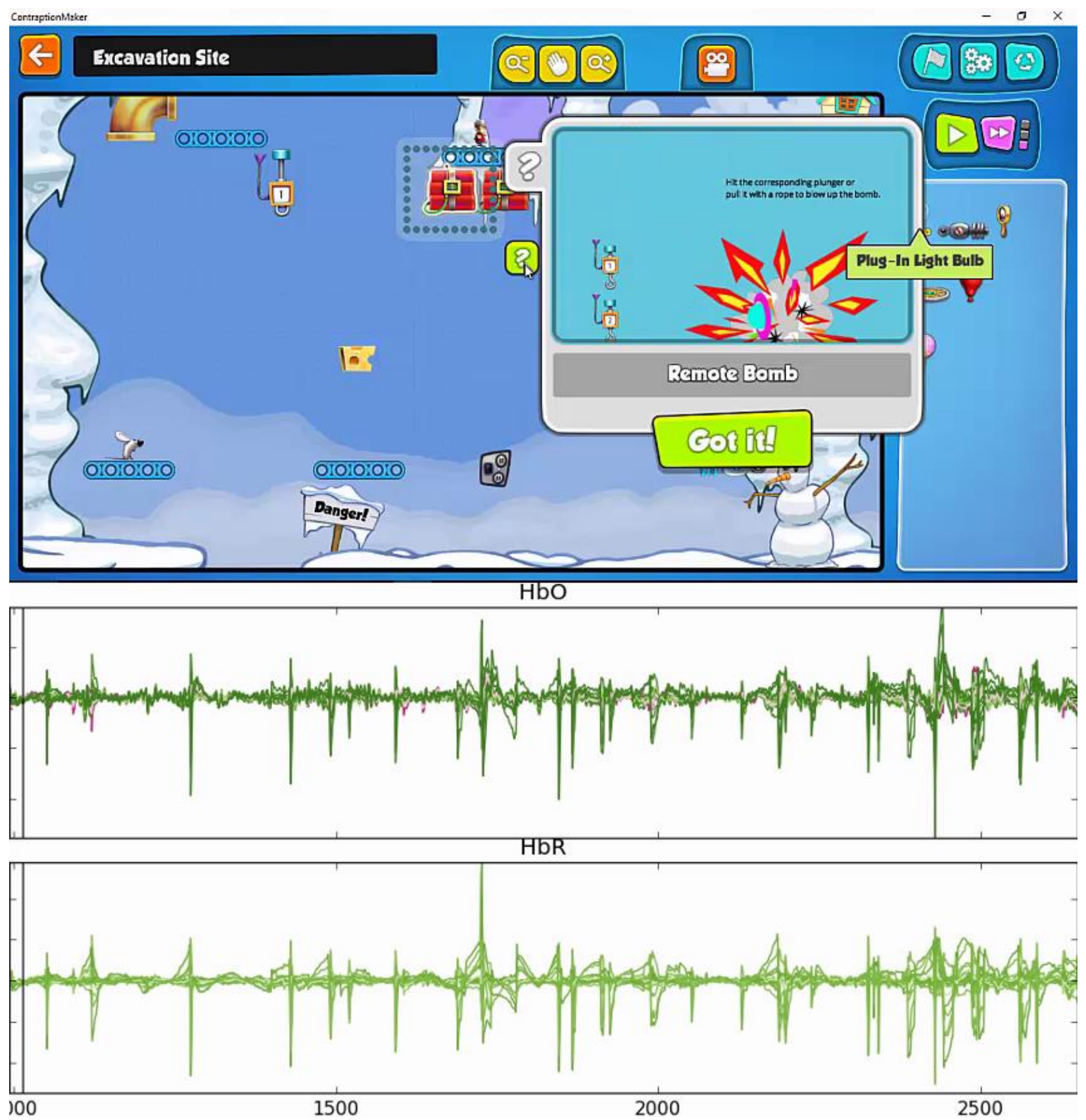

Figure 2: Overlay of video capture from Contraption Maker and processed fNIRS data showing hemodynamic response

The purpose of this paper is not to formally report and discuss specific findings, but in the spirit of discussing what analysis entails, we briefly describe our analysis protocol for this study as it aims to explore an alternate study design.

Perhaps the best descriptor for our research design in this exploratory project is a post-hoc derived event-related design. Essentially, we comb the audio from problem solving and retrospective interviews as well as code the observed behaviors involved in the problem-solving on-screen for specific triggers that indicate a moment of interest. For now, we have targeted 
insight and frustration or giving up moments as our first layer of phenomenon to identify (e.g., "insight" moments triggered by verbal aha, "I knew what to do once I discovered..." in retrospective interviews, or a sudden acceleration/cascading to solution observed in the video data such as when a key connection of pieces results in the rapid solving of a jigsaw puzzle). Having identified these moments per individual across a series of problems solved, we collectively call these the events that would have been pre-scripted in a traditional study design. Though our work is exploratory and deviates from the protocol of traditional blocked and eventrelated design, one advantage to focusing on a concept such as insight this is that previous work has studied neural correlates of insight in simpler problems and so can serve as an opportunity for us to try to validate our methods in this more complex endeavor.

As briefly discussed earlier, between software tools specific to different fNIRS machines and open-source tools (e.g. NIRSLab is specific and proprietary to NIRx Machine), conversion of individual channels of data from source-detector pairs to the oxygenated and deoxygenated hemoglobin data that serve as proxies for neural activity. For example, we have scripts converting our raw data to a format (https://github.com/hazybluedot/pyNIRS) readable by Homer, which is an open-source tool to filter signals and produce the hemodynamic response curves. With a question about neural correlates, we will use multivariate methods that essentially explore what areas reliably co-activate with our stimuli. This is in contrast to univariate methods which can be used to ask questions such as activation in one region compared to another or to explore the effects of a given stimulus on a particular region.

\section{Example 2: Brainstorming Engineering Design Solutions}

Whereas the Contraption Maker study is more closely related to an event related design, this example is more closely related to a block design. Students were given 10 engineering challenges based on Richard Smalley's list of the most pressing issues facing humanity [32] in the next 50 years. Out of the 10 engineering challenges, half required students to consider sustainable design principles when developing solutions. The other 5 engineering challenges did not. The sustainable design requirement for solutions acted as a parameter to the design process. The parameters were developed from previous research on whole systems thinking to enable sustainability in engineering [33]. For example, one question that required students to consider sustainable design principles asked them to "generate as many solutions as possible to heat a house without using mechanical systems." The question directly relates to Richard Smalley's concern for energy and whole system design's use of passive methods for heating and cooling. Students received both blocks of 5 engineering challenges with and without sustainable design requirements. The order in which they received the block was random. Each challenge was displayed on the screen for 60 seconds followed by a rest period of 30 seconds. The timing was based on preliminary studies to ensure neither too much or too little time for the brainstorming sessions. The purpose of the rest period is to normalize activated brain regions before the next task. The time frame for the resting period was chosen because this is double the length of the typical BOLD response experienced from an event onset. Though, by reviewing pilot study data and video recordings, we recognized that students would frequently reflect on their brainstorming performance during the rest period which caused a spike in the cognitive activation in the prefrontal cortex. So, to correct for participants reflecting on the previous task we included simple arithmetic questions every ten seconds. Participants were asked to answer three arithmetic problems between each task. While these arithmetic problems do require brain activation, the region of activation is not the same [34], [35]. Based on prior research, simple 
arithmetic problems are often solved from memory not processing. Students verbally expressed their design solutions during the brainstorming tasks and a researcher tallied the number of responses. Experiments about brainstorming are typically based on the number or novelty of solutions generated. In this study, the number of responses was the main measurement because of its objectivity.

Results of the study could be interpreted using numerous methods aggregating the data. After converting the raw data files to a file format readable by Homer and filtering the data using Homer, the data were averaged across channels (i.e. connected sensors and detectors) because the hypothesis was that requiring thinking about sustainable design principles was cognitively more demanding compared to developing solutions to engineering challenges that did not require this additional consideration. Channels were averaged across the whole prefrontal cortex, split between left and right hemisphere prefrontal cortex, and individually analyzed. The second hypothesis was that students' ability to cognitively manage this additional requirement varied based on years of education. Student participant data were split between freshman engineering students and seniors. Each block (requiring sustainable design consideration or not) was then averaged, creating 1 series of 60 second data. Analysis of variance was run between parameters v. non parameters and freshman v. seniors to examine if the samples were significantly different from each other. The data processing methods and protocol for block related design are considerably easier to implement than event related design because the amount of time for each session within a block is the same. Thus, data analysis is more straightforward compared to an event related design, like the Contraption Maker study which requires post-hoc evaluation to determine the event and assumptions about how much time before and after the event to include in the analysis.

\section{Conclusion}

Better understanding the role of certain brain regions during educational experiments like problem solving and design across a range of subject groups promises to advance teaching and education theory. There are clear and consistent mappings between events at the neural level and events at the behavioral level thus this method can help triangulate other data sources engineering education researchers are already collecting. For example, fNIRS can help answers questions like what degree of accuracy can observed behaviors (i.e. insight or giving up) be classified using only participants' BOLD response and what are the neural correlates (i.e. associated regions) of insight and giving up during extended problem solving. What's more, fNIRS can contribute to advancing understanding of cognitive flexibility and cognitive load theory, providing a step towards new scientific discovery.

Likely of interest to engineering education researchers is the prefrontal cortex due to its acknowledged role in linking cognition, reasoning, and working memory. While this paper was to introduce the imaging tool, our current studies described in the paper are leading to confirmation that fNIRs measured prefrontal cortex activity can be useful in distinguishing changes in cognitive state and across subject types. Important to remember, fNIRs hemodynamic signals are subject to bio-physical noise and instrument noise and raw data must be processed before analysis. Many data analysis methods exist (e.g., the slope of the BOLD response, pattern detection, general linear model, multivariate techniques). The appropriateness of the data analysis approach heavily relies on the type of experimental design. 
Ultimately, bridging neuroscience to engineering education is an area that requires the integrated understanding of both disciplines. At the same time, the merging of disciplines offers to advance cognitive neuroscience more generally by addressing the data collection challenges that arise when extending methods from task-oriented problems to more cognitively complex design challenges that often lack a standardized event onset. The intent of this paper was to communicate details to help readers understand what is involved with fNIRS and the methods and procedures that are required. We see opportunity in measuring the cognitive demand and localization of brain activation using fNIRS. Benefits, such as, offering a new data source, the ability to triangulate other measured data, and opportunity to advance theoretical understanding seems to outweigh the learning curve associated with the measurement tool and nuances of the experimental design to successfully integrate fNIRS into engineering education research.

\section{References}

[1] "Home," NSF: Understanding the Brain. [Online]. Available: https://media.science360.gov/. [Accessed: 20-Jan-2017].

[2] M. W. Eysenck and M. T. Keane, Cognitive Psychology: A Student's Handbook. Psychology Press, 2015.

[3] W. R. Shadish, T. D. Cook, and D. T. Campbell, Experimental and Quasi-experimental Designs for Generalized Causal Inference. Houghton Mifflin, 2002.

[4] I. P. Pavlov, Conditioned Reflexes: An Investigation of the Physiological Activity of the Cerebral Cortex. Oxford Univ.Press: Humphrey Milford, 1927.

[5] M. Coltheart, "Assumptions and methods in cognitive neuropsychology," Handb. Cogn. Neuropsychol. What Deficits Reveal Hum. Mind, pp. 3-21, 2001.

[6] A. Karmiloff-Smith, Beyond Modularity: A Developmental Perspective on Cognitive Science. MIT Press, 1995.

[7] J. E. Ormrod, Human Learning. Pearson/Merrill Prentice Hall, 2008.

[8] H. V. Carter and H. Gray, English: Principal fissures and lobes of the cerebrum viewed laterally. 1858.

[9] "Brodmann area," Wikipedia. 09-Nov-2016.

[10] K. Brodmann, Brodmann's: Localisation in the Cerebral Cortex. Springer Science \& Business Media, 2007.

[11] "Integrative Strategies for Understanding Neural and Cognitive Systems (NSF-NCS) (nsf17519) | NSF - National Science Foundation.” [Online]. Available: https://www.nsf.gov/pubs/2017/nsf17519/nsf17519.htm. [Accessed: 16-Jan-2017].

[12] K. Jablokow et al., "Exploring the Impact of Cognitive Style and Academic Discipline on Design Prototype Variability," presented at the 2014 ASEE Annual Conference \& Exposition, 2014, p. 24.587.1-24.587.13.

[13] C. L. Dym, A. M. Agogino, O. Eris, D. D. Frey, and L. J. Leifer, "Engineering Design Thinking, Teaching, and Learning," J. Eng. Educ., vol. 94, no. 1, pp. 103-120, Jan. 2005.

[14] A. Girouard et al., "From brain signals to adaptive interfaces: using fNIRS in HCI," in Brain-Computer Interfaces, Springer, 2010, pp. 221-237.

[15] M. P. Uysal, "Evaluation of learning environments for object-oriented programming: measuring cognitive load with a novel measurement technique," Interact. Learn. Environ., vol. 0, no. 0, pp. 1-20, May 2015. 
[16] K. Alexiou, T. Zamenopoulos, and S. Gilbert, "Imaging the Designing Brain: A Neurocognitive Exploration of Design Thinking," in Design Computing and Cognition '10, J. S. Gero, Ed. Springer Netherlands, 2011, pp. 489-504.

[17] R. C. Dalton, C. Hölscher, and H. J. Spiers, "Navigating Complex Buildings: Cognition, Neuroscience and Architectural Design," in Studying Visual and Spatial Reasoning for Design Creativity, J. S. Gero, Ed. Springer Netherlands, 2015, pp. 3-22.

[18] M. Boccia, L. Piccardi, L. Palermo, R. Nori, and M. Palmiero, "Where do bright ideas occur in our brain? Meta-analytic evidence from neuroimaging studies of domain-specific creativity," Cognition, p. 1195, 2015.

[19] M. A. Lindquist and others, "The statistical analysis of fMRI data," Stat. Sci., vol. 23, no. 4, pp. 439-464, 2008.

[20] M. Borrego, "Conceptual Difficulties Experienced by Trained Engineers Learning Educational Research Methods," J. Eng. Educ., vol. 96, no. 2, pp. 91-102, Apr. 2007.

[21] M. Borrego, E. P. Douglas, and C. T. Amelink, "Quantitative, Qualitative, and Mixed Research Methods in Engineering Education," J. Eng. Educ. Wash., vol. 98, no. 1, pp. 5366, Jan. 2009.

[22] S. A. Huettel, A. W. Song, and G. McCarthy, Functional Magnetic Resonance Imaging. Sinauer Associates, Incorporated, 2014.

[23] J. Grohs, D. Maczka, M. Soledad, and K. Bagalkotkar, "Exploring the Feasibility of an Educational Computer Game as a Novel Means of Assessing Problem Solving Competencies," 2016.

[24] K. Duncker, "On problem-solving.," Psychol. Monogr., vol. 58, no. 5, p. i-113, 1945.

[25] A. H. Danek, J. Wiley, and M. Öllinger, "Solving Classical Insight Problems Without Aha! Experience: 9 Dot, 8 Coin, and Matchstick Arithmetic Problems," J. Probl. Solving, vol. 9, no. 1, Feb. 2016.

[26] C. J. Atman and K. M. Bursic, "Verbal Protocol Analysis as a Method to Document Engineering Student Design Processes," J. Eng. Educ., vol. 87, no. 2, pp. 121-132, Apr. 1998.

[27] M. Koro-Ljungberg, E. P. Douglas, D. Therriault, Z. Malcolm, and N. McNeill, "Reconceptualizing and decentering think-aloud methodology in qualitative research," Qual. Res., vol. 13, no. 6, pp. 735-753, Dec. 2013.

[28] K. A. Ericsson and H. A. Simon, "Verbal reports as data.," Psychol. Rev., vol. 87, no. 3, pp. 215-251, 1980.

[29] A. Sassaroli and S. Fantini, "Comment on the modified Beer-Lambert law for scattering media," Phys. Med. Biol., vol. 49, no. 14, p. N255, 2004.

[30] Q. Chen, J. Zhao, C. H. Fang, and D. Wang, "Feasibility study on identification of green, black and Oolong teas using near-infrared reflectance spectroscopy based on support vector machine (SVM)," Spectrochim. Acta. A. Mol. Biomol. Spectrosc., vol. 66, no. 3, pp. 568574, Mar. 2007.

[31] S. Tak and J. C. Ye, "Statistical analysis of fNIRS data: A comprehensive review," NeuroImage Amst., vol. 85, pp. 72-91, Jan. 2014.

[32] R. Smalley, "Top ten problems of humanity for next 50 years," 2003.

[33] J. L. Blizzard and L. E. Klotz, “A framework for sustainable whole systems design," Des. Stud., vol. 33, no. 5, pp. 456-479, Sep. 2012.

[34] T. Dresler et al., "Arithmetic tasks in different formats and their influence on behavior and brain oxygenation as assessed with near-infrared spectroscopy (NIRS): a study involving 
primary and secondary school children," J. Neural Transm., vol. 116, no. 12, p. 1689, Dec. 2009.

[35] H. Meiri et al., "Frontal lobe role in simple arithmetic calculations: An fNIR study," Neurosci. Lett., vol. 510, no. 1, pp. 43-47, Feb. 2012. 